\title{
Capacidade funcional de idosos residentes em zona urbana
}

Functional capacity of elderly residents in urban area

Capacidad fucional de ancianos residentes en zona urbana

\section{Joana Darc Chaves Cardoso ${ }^{\mathrm{I}}$, Adriana Delmondes de Oliveira ${ }^{\mathrm{II}}$, Carla Rafaela Teixeira Cunha ${ }^{\mathrm{III}}$, Kátia Moreira da Silva ${ }^{\mathrm{IV}}$}

Resumo: Objetivo: analisar a capacidade funcional dos idosos residentes em zona urbana. Método: estudo transversal, desenvolvido no município de Cuiabá- Mato Grosso. A amostra foi de 573 idosos. Os dados foram analisados por meio de estatística descritiva. Resultados: Houve predomínio de idosos na faixa etária de 60 a 69 anos $(45,9 \%)$ e que residiam com outras pessoas $(89,5 \%)$. A maioria dos homens era casada ou tinha companheira $(72,8)$ e as mulheres eram viúvas (43,6\%). Os homens idosos eram mais independentes para realizar suas atividades diárias $(72,4 \%)$, enquanto as mulheres eram mais dependentes (41,7\%). Conclusão: considera-se relevante incorporar a avaliação da Capacidade Funcional na consulta de enfermagem ao idoso, com a finalidade de prevenir e minimizar os prejuízos da dependência funcional.

Descritores: Idoso; Saúde do idoso; Área urbana

\footnotetext{
Abstract: Aim: to analyze the functional capacity of elderly people living in urban areas. Method: a cross-sectional study, developed in the city of Cuiabá-Mato Grosso. The sample was 573 elderly people. Data were analyzed through descriptive statistics. Results: There was a predominance of elderly people in the age group of 60 to 69 years-old

${ }^{\text {I }}$ Enfermeira. Docente da Faculdade de Enfermagem da Universidade Federal de Mato Grosso (UFMT), doutoranda do Programa de Pós-Graduação em Enfermagem da UFMT. Cuiabá-MT, Brasil. E-mail: joana-qtal@hotmail.com ORCID iD: https://orcid.org/0000-0003-1989-4043

II Enfermeira. Docente da Faculdade FASIPE-CPA. Mestre em Enfermagem pela UFMT, doutoranda do Programa de Pós-Graduação em Enfermagem da UFMT. Cuiabá-MT, Brasil. E-mail: drydelondes@gmail.com ORCID iD: https://orcid.org/0000-0002-0100-413X

III Enfermeira. Docente da Faculdade de Enfermagem UFMT, doutoranda do Programa de Pós-Graduação em Enfermagem UFMT. Cuiabá-MT, Brasil. E-mail: ca_rafa_enf@hotmail.com ORCID iD: https://orcid.org/0000-0001-7084-221X

IV Enfermeira. Mestre em Enfermagem pela UFMT. Residente do Programa de Residência Multiprofissional em Saúde do Adulto e do Idoso da Universidade Federal de Rondonópolis. Rondonópolis-MT, Brasil. katiakawam@hotmail.com ORCID iD: https://orcid.org/0000-0002-7679-0040
} 
(45.9\%) who lived with other people (89.5\%). Most men were married or had a partner (72.8\%) and women were widows (43.6\%). Elderly men were more independent to perform their daily activities $(72.4 \%)$ while women were more dependent (41.7\%). Conclusion: it is considered relevant to incorporate the Functional Capacity evaluation in the nursing consultation to the elderly, with the purpose of preventing and minimizing the losses of functional dependence.

Descriptors: Elderly; Elderly health; Urban area

Resumen: Objetivo: analizar la capacidad funcional de los ancianos residentes en zona urbana. Método: estudio transversal, desarrollado en el municipio de Cuiabá - Mato Grosso. La muestra fue constituida por 573 ancianos. Los datos fueron analizados por medio de estadística descriptiva. Resultados: Hubo predominio de ancianos en el grupo de edad de 60 a 69 años (45,9\%), que residían con otras personas (89,5\%). La mayoría de los hombres estaban casados o tenían compañera $(72,8)$ y las mujeres eran viudas $(43,6 \%)$. Los hombres mayores eran más independientes para realizar sus actividades diarias (72,4\%), mientras que las mujeres eran más dependientes (41,7\%). Conclusión: se considera relevante incorporar la evaluación de la Capacidad Funcional en la consulta de enfermería al anciano, con la finalidad de prevenir y minimizar los problemas de dependencia funcional.

Descriptores: Ancianos; Salud del anciano; Área urbana

\section{Introdução}

O envelhecimento populacional há alguns anos, tem sido uma realidade vivenciada nos países em desenvolvimento. O último censo realizado no Brasil em 2010 mostrou que os idosos já somam mais de 20 milhões de pessoas, representando $10,8 \%$ da população geral. ${ }^{1}$

Esse envelhecimento é acompanhado do aumento da ocorrência de prevalência das Doenças Crônicas Não Transmissíveis (DCNT), principalmente as doenças cardiovasculares, respiratórias, neoplasias, diabetes mellitus e do trato gastrintestinal. ${ }^{2}$ Tais doenças podem interferir na capacidade funcional (CF) do idoso, comprometendo sua autonomia e independência, levando a incapacidades e, consequentemente, a dependência funcional..$^{3-4}$

A CF é a interação entre a capacidade física e mental que o idoso possui para exercer atividades consideradas importantes para si e para sua sobrevivência, como as Atividades de 
Vida Diária (AVD) e as Atividades Instrumentais de Vida Diária (AIVD). ${ }^{2}$ As AVD são atividades básicas como alimentar-se, andar e vestir-se e as AIVD são as atividades mais complexas que indicam uma independência maior em tomar medicações, administrar seu dinheiro, pegar uma condução, preparar sua própria refeição. ${ }^{5-6}$

Já a incapacidade funcional é a dificuldade que o idoso possui em realizar suas atividades do cotidiano decorrente de alguma limitação física ou mental, o que pode implicar na dependência de cuidados de seus familiares, comunidade e Instituições de Longa Permanência para Idosos (ILPI). ${ }^{7}$

O declínio da CF é um dos principais problemas que pode afetar a pessoa idosa. ${ }^{3,5}$ Estudos internacionais e nacionais sobre essa temática tem explorado alguns aspectos, principalmente fatores que levam o idoso a tornar-se incapaz..$^{8-9}$ Os nacionais têm direcionado o seu foco na perda da CF, e têm constatado que os principais fatores associados à incapacidade funcional são, idade avançada, sexo feminino, baixa renda, baixa escolaridade e DCNT.7-8,10 Os estudos internacionais tem constatado que os principais fatores associados a perda da CF são idade avançada, uso de cinco ou mais medicamentos e depressão. ${ }^{11-12}$

Investigação realizada na região metropolitana de Belo Horizonte com 1.624 idosos constatou que 19,6\% eram dependentes para realizar alguma AIVD e 16,2\% tinham alguma dificuldade em realizar uma ou mais AVD. ${ }^{8}$ Em Porto Alegre, pesquisa realizada com 671 idosos identificou que $15,5 \%$ necessitavam de ajuda para as AVD e $26,1 \%$ precisavam de ajuda para realizar pelo menos uma AIVD. ${ }^{3}$

A CF é apontada na literatura como um dos principais componentes da saúde do idoso, configurando um novo paradigma de saúde para a população que envelhece. Assim, o indicador de saúde mais importante passa a ser o grau de CF da pessoa e não mais a presença de morbidades $^{13}$ pois, mesmo na presença dessas, ${ }^{9}$ o idoso pode manter suas atividades do cotidiano. ${ }^{14}$ 
Considerando a importância de promover a saúde do idoso, o Ministério da Saúde (MS) assumiu que o principal problema que pode afeta-la é a perda da capacidade de realizar suas atividades do cotidiano. Nesse sentido, criou-se em 2010 a Política Nacional de Saúde da Pessoa Idosa (PNSPI) com objetivo de priorizar a promoção, manutenção e reabilitação da CF. ${ }^{2}$ Assim, para sustentar as ações previstas nesta política, bem como para eleger prioridades pelos gestores, alocar recursos e planejar a assistência aos idosos, mantendo-os ativos na sociedade, torna-se indispensável conhecer suas necessidades de saúde, assim como sua capacidade de realizar as atividades do cotidiano. ${ }^{14}$ Diante ao exposto, questiona-se: como se apresenta a capacidade funcional de idosos residentes em zona urbana? Para responder a esse questionamento, teve-se por objetivo analisar a Capacidade Funcional dos idosos residentes em zona urbana.

\section{Método}

O presente artigo utiliza dados extraídos do estudo intitulado "Condições de saúde autorreferidas da população idosa do município de Cuiabá”, que analisou as condições de saúde dessa população. ${ }^{15}$ De acordo com o último censo, o município de Cuiabá possui 551.098 habitantes, destes 44.817 são idosos. ${ }^{16}$

Trata-se de um estudo transversal, desenvolvido com pessoas de 60 anos ou mais que residem na zona urbana. Foram excluídas aquelas que residiam em ILPI, hospitais, presídios, albergues e casas de apoio.

A amostra foi definida por meio do cálculo estatístico para populações finitas. Considerouse um coeficiente de confiança de 95\%, erro de amostragem de 5\% e um valor de proporção de 0,5 ( $\mathrm{p}=0,5)$. Dessa forma, a partir da amostragem por conglomerado, a amostra final foi de 573 idosos. 
Os dados foram coletados no domicílio dos idosos, no período de dezembro de 2011 a março de 2012 por meio do questionário BOAS (Brazil Old Age Schedule). ${ }^{17}$ Trata-se de uma ferramenta multidimensional que tem sido usada em pesquisas com a população idosa. O questionário é dividido em nove seções, totalizando 75 questões.

Para analisar a CF dos idosos foram utilizadas as seções I (informações gerais do idoso) e IV (AVD). Para avaliar a CF e estabelecer o grau de dependência, considerou-se a capacidade do idoso realizar 12 AVD (sair de casa com transporte, sair curtas distâncias, comer a sua refeição, tomar remédios, vestir-se e despir-se, pentear seus cabelos, caminhar no plano, subir/descer escadas, deitar e levantar, tomar banho, cortar as unhas dos pés, ir ao banheiro em tempo). Estas AVD variavam entre atividades mais simples às mais complexas.

As variáveis estudadas foram as seguintes:

- Sociodemográficas: sexo (masculino/feminino), idade (60-69 anos, 70-79 anos, 80 anos e mais), sabe ler e escrever (sim/não), escolaridade máxima (nenhuma, primário, ginásio ou primeiro grau), estado conjugal (casado(a)/possui companheiro(a), viúvo(a), divorciado(a)/separado(a), nunca se casou), moradores no domicílio (mora sozinho/mora com outras pessoas).

- Grau de dependência: independente (idosos que não apresentam incapacidade ou dificuldade na realização das AVD), dependência leve (apresenta incapacidade ou dificuldade para realizar de uma a três AVD) ou dependência moderada/grave (idosos que apresentam incapacidade ou dificuldade em realizar quatro ou mais AVD). ${ }^{17}$ Pessoa para ajudar nas tarefas diárias (sim, não), pessoa que mais ajuda nas tarefas diárias (esposo (a)/companheiro (a), filho, filha, uma outra pessoa da família, um (a) empregado (a), outra).

Os dados foram processados no programa SPSS 15.0 (Statistical Package for Social Sciences) e foram analisados de forma descritiva, com frequências absolutas e relativas. Esta pesquisa foi aprovada pelo Comitê de Ética em Pesquisa do Hospital Universitário Júlio Müller, 
sob protocolo n $132 / \mathrm{CEP} / \mathrm{HUJM} / 11$ e foram seguidos todos os preceitos éticos da Resolução 466/12, do Conselho Nacional de Saúde.

\section{Resultados}

Participaram do estudo 573 idosos (254 homens e 319 mulheres), com faixa etária entre 60 e 69 anos (45,9\%). Os homens eram casados ou possuíam companheira $(72,8 \%)$ e a maioria das mulheres era viúva (43,6\%). Quanto à escolaridade, 73,5\% dos idosos sabiam ler e escrever, e a escolaridade máxima foi o ensino primário (44,4\%). Idosos que residiam com outras pessoas somaram $89,5 \%$.

Tabela 1- Distribuição dos idosos segundo características demográficas e socioeconômicas, segundo sexo. Cuiabá-MT. 2012.

\begin{tabular}{|c|c|c|c|c|c|c|}
\hline Variável & $\mathbf{n}$ & $\begin{array}{c}\text { Homens } \\
\begin{array}{c}\text { n= } 254 \\
\%\end{array}\end{array}$ & $\mathbf{n}$ & $\begin{array}{c}\text { Mulheres } \\
\text { n=319 } \\
\%\end{array}$ & $\mathbf{n}$ & $\begin{array}{c}\text { Total } \\
\mathbf{n}=573 \\
\%\end{array}$ \\
\hline \multicolumn{7}{|l|}{ Faixa etária } \\
\hline 60 a 69 anos & 118 & 46,5 & 145 & 45,5 & 263 & 45,9 \\
\hline 70 a 79 anos & 82 & 32,3 & 120 & 37,9 & 202 & 35,4 \\
\hline 80 anos ou mais & 53 & 20,5 & 52 & 16,3 & 105 & 18,2 \\
\hline $\mathrm{NS} / \mathrm{NR}^{* *}$ & 03 & 00,8 & 02 & 00,3 & 03 & 00,5 \\
\hline \multicolumn{7}{|l|}{ Estado civil } \\
\hline $\begin{array}{l}\text { Casado(a)/ } \\
\text { Possui companheiro(a) }\end{array}$ & 185 & 72,8 & 124 & 38,9 & 309 & 53,9 \\
\hline Viúvo(a) & 37 & 14,6 & 139 & 43,6 & 176 & 30,7 \\
\hline $\begin{array}{l}\text { Divorciado(a)/ } \\
\text { Separado (a) }\end{array}$ & 23 & 09,1 & 35 & 11,0 & 58 & 10,1 \\
\hline Nunca se casou & 09 & 03,5 & 21 & 06,6 & 30 & 05,2 \\
\hline \multicolumn{7}{|l|}{ Sabe ler e escrever } \\
\hline $\operatorname{Sim}$ & 188 & 74,0 & 233 & 73,0 & 421 & 73,5 \\
\hline Não & 66 & 26,0 & 86 & 27,0 & 152 & 26,5 \\
\hline \multicolumn{7}{|l|}{ Escolaridade completa } \\
\hline Nenhuma & 29 & 15,4 & 40 & 17,2 & 69 & 16,4 \\
\hline Primário & 82 & 43,6 & 105 & 45,1 & 187 & 44,4 \\
\hline $\begin{array}{l}\text { Ginásio ou Primeiro } \\
\text { grau }\end{array}$ & 24 & 12,8 & 31 & 13,3 & 55 & 13,1 \\
\hline Segundo grau & 24 & 12,8 & 33 & 14,2 & 57 & 13,5 \\
\hline Superior & 26 & 13,8 & 22 & 09,4 & 48 & 11,4 \\
\hline
\end{tabular}


7 I Cardoso JDC, Oliveira AD, Cunha CRT, Silva KM

\begin{tabular}{|c|c|c|c|c|c|c|}
\hline $\mathrm{NS} / \mathrm{NR}^{* *}$ & 03 & 01,6 & 02 & 00,9 & 05 & 01,2 \\
\hline \multicolumn{7}{|l|}{ Com quem mora } \\
\hline Sozinho (a) & 26 & 10,2 & 34 & 10,7 & 60 & 10,5 \\
\hline Com outras pessoas & 228 & 89,8 & 91 & 89,3 & 513 & 89,5 \\
\hline
\end{tabular}

Em relação à CF dos idosos, 64,5\% eram independentes, dentre eles 72,4\% eram homens e $58,3 \%$ eram mulheres. A frequência de dependência (leve, moderada/grave) entre os homens foi de $27,6 \%$ e para as mulheres $41,7 \%$ (Tabela 2 ).

Tabela 2- Distribuição da capacidade funcional dos idosos, segundo sexo considerando o grau de dependência. Cuiabá-MT, 2012.

\begin{tabular}{|c|c|c|c|c|c|c|}
\hline Variável & $\mathbf{n}$ & $\begin{array}{c}\text { Homens } \\
\begin{array}{c}\text { n=254 } \\
\%\end{array}\end{array}$ & $\mathbf{n}$ & $\begin{array}{c}\text { Mulheres } \\
\text { n=319 } \\
\%\end{array}$ & $\mathbf{n}$ & $\begin{array}{c}\begin{array}{c}\text { Total } \\
\mathbf{n}=\mathbf{5 7 3} \\
\%\end{array} \\
\end{array}$ \\
\hline \multicolumn{7}{|c|}{ Grau de dependência } \\
\hline Independência & 18 & 72,4 & 186 & 58,3 & 370 & 64,5 \\
\hline Dependência leve & 50 & 19,7 & 105 & 32,9 & 155 & 27,1 \\
\hline $\begin{array}{l}\text { Dependência } \\
\text { moderada/grave }\end{array}$ & 20 & 7,9 & 28 & 8,8 & 48 & 8,4 \\
\hline
\end{tabular}

A maioria dos idosos $(75,7 \%)$ necessita de alguém para auxiliar na realização de suas tarefas diárias, com frequência maior entre os homens (83,5\%). Para os homens, a pessoa que mais ajudava na realização das atividades do cotidiano foram as esposas/companheiras (55,2\%) e, para as mulheres, as filhas $(42,2 \%)$.

Tabela 3- Pessoas que ajudam os idosos nas tarefas diárias, segundo sexo. CuiabáMato Grosso-2012.

\begin{tabular}{ccccccc}
\hline & & Homens & & Mulheres & Total \\
Variável & n & $\mathbf{n}=\mathbf{2 5 4}$ & n & $\mathbf{n}=\mathbf{3 1 9}$ & n & $\mathbf{n}=\mathbf{5 7 3}$ \\
& & $\%$ & & $\%$ & & $\%$ \\
\hline
\end{tabular}

Pessoa para ajudar nas 


\begin{tabular}{|c|c|c|c|c|c|c|}
\hline \multicolumn{7}{|l|}{ tarefas diárias } \\
\hline Sim & 222 & 83,5 & 212 & 69,6 & 434 & 75,7 \\
\hline Não & 32 & 16,5 & 107 & 30,4 & 139 & 24,3 \\
\hline \multicolumn{7}{|l|}{$\begin{array}{l}\text { Pessoa que mais ajuda } \\
\text { nas tarefas diárias }\end{array}$} \\
\hline Esposo/ Companheiro(a) & 117 & 55,2 & 31 & 13,9 & 148 & 34,0 \\
\hline Filho & 09 & 4,2 & 17 & 7,6 & 26 & 6,0 \\
\hline Filha & 36 & 17,0 & 94 & 42,2 & 130 & 29,9 \\
\hline $\begin{array}{l}\text { Uma outra pessoa da } \\
\text { família }\end{array}$ & 16 & 7,5 & 42 & 18,8 & 58 & 13,3 \\
\hline Um(a)empregado(a) & 23 & 10,8 & 33 & 14,8 & 56 & 12,9 \\
\hline
\end{tabular}

\section{Discussão}

Os resultados deste estudo contribuem para ampliação do conhecimento sobre a CF e as necessidades de cuidado dos idosos que residem em zona urbana e apontam aspectos que devem ser abordados na assistência a essa população. As características sociodemográficas e a CF dos idosos apontam uma maioria de idosos jovens, homens casados, mulheres viúvas, baixa escolaridade, residem com alguém, são independentes, entretanto, precisam de auxílio na realização das AVD.

O fato dos idosos da amostra serem mais jovens pode ser explicado pelo recente envelhecimento da população no Brasil..,18-19 Os “idosos jovens” tendem a apresentar maior independência na realização das AVD quando comparado a idosos mais velhos, pois com o avançar da idade ocorre um declínio fisiológico que pode comprometer sua autonomia e independência. ${ }^{20}$

A maior proporção de homens casados ou que possuem companheiras e de mulheres viúvas é um resultado consistente com de outros estudos. ${ }^{20-21}$ Isso pode ocorrer pelo fato de os homens ao se divorciarem ou ficarem viúvos constituírem novo casamento, já as mulheres quando vivenciam tal situação tendem a permanecerem viúvas. Somado a isso, há de se considerar a maior sobrevida das mulheres, aumentando a chance de continuarem viúvas. ${ }^{21}$ 
Evidências tem constatado que a viuvez pode influenciar negativamente a CF dos idosos. Investigação desenvolvida na cidade de Guarapuava, no Paraná, com o objetivo de analisar a CF de 359 idosos, identificou associação da viuvez com o grau de dependência das idosas. ${ }^{18}$

A baixa escolaridade encontrada nesse estudo talvez seja consequência de os idosos terem vivido em uma época em que a população brasileira se concentrava em zona rural, a educação era priorizada para os homens e ou pessoas com condições socioeconômicas mais favoráveis. ${ }^{3,19,21}$

A CF está diretamente ligada ao grau de escolaridade dos idosos, pois pode influenciar na realização de suas AIVD, como o uso de transporte público, tomar medicamentos e gerir suas finanças, ou seja, quanto menor a escolaridade, maior o nível de dependência. ${ }^{20}$

A corresidência do idoso ocorre por fatores culturais e econômicos, pois com o avançar da idade há um comprometimento maior de sua renda, com medicamentos, saúde e alimentação. Eles continuam, muitas vezes, a ajudar na renda familiar ou são os principais provedores. ${ }^{22}$

Observa-se, quanto a $\mathrm{CF}$, que a maioria dos idosos era independente, com frequência maior entre os homens. Esse resultado é semelhante ao de outro estudo ${ }^{18}$ que encontrou homens mais independentes que as mulheres.

A incapacidade funcional é um fenômeno que difere entre os gêneros. Algumas possíveis explicações podem ser atribuídas a essa diferença, como o fato de as mulheres apresentarem maior sobrevida, maior prevalência de problemas crônicos de saúde e elevados níveis de dependência, que podem comprometer sua independência e autonomia. ${ }^{18,20-22}$ Homens e mulheres são influenciados não somente pelo fator biológico, mas também pelo contexto sóciohistórico-cultural, que definirá o seu padrão de comportamento, determinando um envelhecimento bem-sucedido ou não. ${ }^{23}$

Outro achado importante é o fato de os homens requererem maior auxílio de esposas ou companheiras para desenvolver suas tarefas diárias. Evidências apontam que isso pode 
representar maior apoio emocional, caracterizando o companheirismo no cuidado a saúde. Diferentemente, as mulheres ao permanecerem viúvas, por sua vez, recorrem à filha que desempenha um papel importante na vida social e afetiva das idosas para auxilia-las no cuidado. ${ }^{22-21}$

\section{Conclusão}

Os dados desta investigação permitiram verificar a CF da população idosa residente em zona urbana. A amostra desse estudo tinha faixa etária entre 60 e 69 anos, os homens eram casados ou possuíam companheiras, enquanto as mulheres eram viúvas. A maioria dos idosos residia com outras pessoas, sabia ler e escrever e possuía o ensino primário.

Verificou-se que os homens idosos eram mais independentes para a realização de suas atividades diárias e, quando necessitavam de ajuda, as esposas ou companheiras que exerciam esse papel. Já as mulheres eram mais dependentes e as filhas eram quem auxiliavam nas atividades cotidianas.

Os achados deste estudo reforçam a necessidade dos profissionais de saúde, especialmente aqueles que atuam na Estratégia de Saúde da Família, monitorar a CF dos idosos, tendo em vista os prejuízos que a dependência funcional pode trazer para a vida e à saúde dessa população, de seus familiares e para o sistema de saúde. Essas informações são importantes ainda para o planejamento do cuidado a saúde e considera-se fundamental incorporar a avaliação da CF na consulta de enfermagem ao idoso, com a finalidade de prevenir e minimizar os prejuízos da dependência funcional.

Algumas limitações podem ser citadas, dentre elas que o estudo foi realizado em uma capital da região Centro-Oeste do Brasil, que apresenta diferenças socioculturais e econômicas das demais regiões do país; a população estudada se limita a idosos residentes na comunidade, consequentemente, não reflete a realidade de pessoas residentes em ILPI. 


\section{Referências}

1. Instituto Brasileiro de Geografia e Estatística (IBGE). Censo demográfico 2010. Resultado da amostra: características da população. 2014.

2. Ministério da Saúde (BR), Secretaria de Atenção à Saúde, Departamento de Ações Programáticas e Estratégicas, Área Técnica Saúde do Idoso. Atenção à saúde da pessoa idosa e envelhecimento. Brasília(DF): Ministério da Saúde; 2010.

3. Pereira GN, Bastos GAN, Del Duca GF, Bos AJG. Indicadores demográficos e socioeconômicos associados à incapacidade funcional em idosos. Cad Saúde Pública [Internet]. 2012 [acesso em 2017 jun 10];28(11):2035-42. Disponível em: https://www.scielosp.org/pdf/csp/2012.v28n11/2035-2042/pt

4. Pinto AH, Lange C, Pastore CA, Lhano PMP, Castro DP, Santos F. Capacidade funcional para atividades de vida diária de idosos da Estratégia de Saúde da Família da zona rural. Ciênc Saúde Colet [Internet]. 2016 [acesso em 2018 jan 15];21(11):3545-55. Disponível em: https://www.scielosp.org/scielo.php?pid=S1413-81232016001103545\&script=sci_arttext doi: 10.1590/1413812320152111.22182015

5. Den Ouden MEM, Schuurmans MJ, Mueller-Schotte S, Van der Schouw YT. Identification of high-risk individuals for the development of disability in activities of daily living. A ten-year follow-up study. Exp Gerontol [Internet]. 2013 [acesso em 2018 jan 15];48(4):437-43. Disponível em: https://www.sciencedirect.com/science/article/pii/S0531556513000338?via\%3Dihub doi: 10.1016/j.exger.2013.02.002

6. Carvalho IS, Lima Neto AV, Silva BCO, Nunes VMA, Alchieri JC. Avaliação das atividades básicas e instrumentais de vida diária de idosos participantes de grupos de convivência. Rev Pesqui Cuid Fundam [Internet]. 2014 [acesso em 2017 maio 05];6(2):607-17. Disponível em: http://www.seer.unirio.br/index.php/cuidadofundamental/article/view/3063/pdf_1250 doi: 10.9789/2175$5361.2014 \mathrm{v} 6 \mathrm{n} 2 \mathrm{p} 607$

7. Gavasso WC, Beltrame V. Capacidade funcional e morbidades referidas: uma análise comparativa em idosos. Rev Bras Geriatr Gerontol [Internet]. 2017 [acesso em 2018 jan 05]; 20(3):399-409. Disponível em:http:/www.scielo.br/pdf/rbgg/v20n3/pt_1809-9823-rbgg-20-03-00398.pdf $\quad$ doi: 10.1590/198122562017020.160080

8. Fialho CB, Lima-Costa MF, Giacomin KC, Loyola Filho AI. Capacidade funcional e uso de serviços de saúde por idosos da Região Metropolitana de Belo Horizonte, Minas Gerais, Brasil: um estudo de base populacional. Cad Saúde Pública [Internet]. 2014 [acesso em 2018 jun 22];30(3):599-610. Disponível em: https://www.scielosp.org/scielo.php?pid=S0102-311X2014000300599\&script=sci_arttext\&tlng=pt doi: 10.1590/0102-311X00090913 
9. Virtuoso Júnior JS, Martins CA, Roza LB, Paulo TRS, Ribeiro MCL, Tribess S. Prevalência de incapacidade funcional e fatores associados em idosos. Texto \& Contexto Enferm [Internet]. 2015 [acesso em 2018 jan 10];24(2):521-9. Disponível em: http://www.scielo.br/pdf/tce/v24n2/pt_0104-0707-tce-24-0200521.pdf doi: 10.1590/0104-07072015001652014

10. Wang H, Chen K, Pan Y, Jing F, Liu H. Associations and impact factors between living arrangements and functional disability among older Chinese adults. PLoS One [Internet]. 2013 [acesso em $2017 \mathrm{dez}$ 20];8(1):e53879. Disponível em: http://journals.plos.org/plosone/article?id=10.1371/journal.pone.0053879 doi: 10.1371/journal.pone.0053879

11. Connolly D, Garvey J, McKee G. Factors associated with ADL/IADL disability in community dwelling older adults in the Irish longitudinal study on ageing (TILDA). Disabil Rehabil [Internet]. 2017 [acesso em $2017 \quad \operatorname{dez} 20] ; 39(8): 809-16 . \quad$ Disponível em: https://www.tandfonline.com/doi/abs/10.3109/09638288.2016.1161848 doi: 10.3109/09638288.2016.1161848

12. Ogata S, Hayashi C, Sugiura K, Hayakawa K. Associations between depressive state and impaired higher-level functional capacity in the elderly with long-term care requirements. PloS One [Internet]. 2015 [acesso em 2017 dez 20];10(6):1-10. Disponível em: https://journals.plos.org/plosone/article?id=10.1371/journal.pone.0127410 doi: 10.1371/journal.pone.0127410

13. Campolina AG, Adami F, Santos JLF, Lebrao ML. A transição de saúde e as mudanças na expectativa de vida saudável da população idosa: possíveis impactos da prevenção de doenças crônicas. Cad Saúde Pública [Internet]. 2013 [acesso em 2018 jun 23];29(6):1217-29. Disponível em: https://www.scielosp.org/pdf/csp/2013.v29n6/1217-1229/pt

14. Brito KQD, Menezes TN, Olinda RA. Incapacidade funcional: condições de saúde e prática de atividade física em idosos. Rev Bras Enferm [Internet]. 2016 [acesso em 2017 dez 15];69(5):773-80. Disponível em: http://www.scielo.br/scielo.php?pid=S003471672016000500825\&script=sci_arttext\&tlng=pt doi: 10.1590/0034-7167.2016690502

15. Cardoso JDC, Azevedo RCS, Reiners AAO, Louzada CV, Espinosa MM. Autoavaliação de saúde ruim e fatores associados em idosos residentes em zona urbana. Revista Gaúch Enferm [Internet]. 2014 [acesso em 2019 abr 29];35(4):35-41. Disponível em: https://www.seer.ufrgs.br/RevistaGauchadeEnfermagem/article/view/46916

16. Instituto Brasileiro de Geografia e Estatística (IBGE). Primeiros dados do censo 2010. Dados Mato Grosso. 2010.

17. Veras R, Dutra S. Perfil do idoso brasileiro: questionário BOAS [Internet]. 2008 [acesso em 2018 mar 13]. Rio de Janeiro: UnATI; 2008. Disponível em: http://www.crde-unati.uerj.br/liv_pdf/perfil.pdf 
18. Pilger G, Menon UM, Mathias TAF. Capacidade funcional de idosos atendidos em unidades básicas de saúde do SUS. Rev Bras Enferm [Internet]. 2013 [acesso em 2017 mar 21];66(6):907-13. Disponível em: http://www.redalyc.org/pdf/2670/267029915015

19. Porciúncula RCR, Carvalho EF, Barreto KML, Leite VMM. Perfil sócio epidemiológico e autonomia de longevos em Recife-PE, Nordeste do Brasil. Rev Bras Geriatr Gerontol [Internet]. 2014 [acesso em 2018 maio 14];17(2):315-25. Disponível em: http://www.scielo.br/scielo.php?script=sci_arttext\&pid=S180998232014000200315

20. Berlezi EM, Farias AM, Dallazen F, Oliveira KR, Pillatt AP, Forte CK. Como está a capacidade funcional de idosos residentes em comunidades com taxa de envelhecimento populacional acelerado? Rev Bras Geriatr Gerontol [Internet]. 2016 [acesso em 2017 jul 13]; 19(4):643-52. Disponível em: http://www.scielo.br/pdf/rbgg/v19n4/pt_1809-9823-rbgg-19-04-00643.pdf/ 10.1590/180998232016019.150156

21. Santos SG, Cunha ICKO. Avaliação da capacidade funcional de idosos para desempenho das atividades instrumentais da vida diária: um estudo na atenção básica em saúde. Rev Enferm Cent-Oeste Min [Internet]. 2013[acesso em 2017 jul 13];3(3):820-82. Disponível em: http://www.seer.ufsj.edu.br/index.php/recom/article/view/421. doi: http://dx.doi.org/10.19175/recom.v0i0.421

22. Stamm B, Leite MT, Hildebrandt LM, Kirchner RM, Girardon-Perlini NMO, Beuter M. Cognition and functional capacity of elderly people who live alone and with relatives. Rev Baiana Enferm [Internet]. 2017 [acesso em 2018 abr 19];31(2):e17407. Disponível em: https://www.researchgate.net/profile/marines_leite/publication/320611123_cognition_and_functional_cap acity_of_elderly_people_who_live_alone_and_with_relatives/links/59f47397458515547c21820a/cognitionand-functional-capacity-of-elderly-people-who-live-alone-and-with-relatives.pdf doi: 10.18471/rbe.v31i2.17407

23. Mansur AP, Favarato D. Mortalidade por doenças cardiovasculares em mulheres e homens nas cinco regiões do Brasil, 1980-2012. Arq Bras Cardiol [Internet]. 2016 [acesso em 2018 fev 08];107(2). Disponível em: http://www.scielo.br/pdf/abc/2016nahead/pt_0066-782X-abc-20160102.pdf doi: 10.5935/abc.20160102

\author{
Autor correspondente \\ Joana Darc Chaves Cardoso \\ E-mail: joana-qtal@hotmail.com \\ Endereço: Avenida Fernando Corrêa, 2367, Bairro Boa Esperança, Cuiabá-MT. \\ CEP: 78068-600
}

\title{
Contribuições de Autoria
}


1 - Joana Darc Chaves Cardoso

Pesquisadora responsável pela concepção, planejamento, execução do projeto de pesquisa, redação e revisão crítica do estudo.

2 - Adriana Delmondes de Oliveira

Autora participou da redação e revisão crítica do manuscrito.

3 - Carla Rafaela Teixeira Cunha

Autora participou da redação e revisão crítica do manuscrito.

4 - Kátia Moreira da Silva

Autora participou da redação e revisão crítica do manuscrito.

\section{Como citar este artigo}

Cardoso JDC, Oliveira AD, Cunha CRT, Silva KM. Capacidade funcional de idosos residentes em zona urbana. Rev. Enferm. UFSM. 2019 [Acesso em: Ano Mês Dia];vol e2:1-10. DOI:https://doi.org/10.5902/21797692340954 